\title{
Predicting invasiveness and disease-specific survival in upper tract urothelial carcinoma: identifying relevant clinical tumour characteristics
}

\author{
Camilla Malm ${ }^{1,2}$ (D) Alexandra Grahn ${ }^{1,3}\left(\mathbb{D} \cdot\right.$ Georg Jaremko $^{1,4}(\mathbb{D}) \cdot$ Bernhard Tribukait $^{1} \cdot$ Marianne Brehmer $^{5}$
}

Received: 1 January 2019 / Accepted: 4 April 2019 / Published online: 23 April 2019

(c) The Author(s) 2019

\begin{abstract}
Purpose The aim of this prospective study was to identify the tumour characteristics that are associated with invasiveness and those that are relevant for disease-specific survival (DSS) in upper tract urothelial carcinoma, UTUC.

Methods From a prospective consecutive cohort of patients with suspicion of UTUC, those who were diagnosed with UTUC using URS prior to rNU between 2005 and 2012 were included. Tumour characteristics were analysed for prediction of invasiveness and association with DSS. Stages were categorised as superficial (pTa-1 and CIS only) or invasive ( $\geq$ pT2). Tumours were graded according to WHO 1999 classification. DSS was analysed regarding possible association with stage, grade, size, multifocality, location, ploidy and rate of proliferation. Associations were tested using Fisher's exact test, Pearson Chi-square or Cox's regression. Kaplan-Meier survival curves were constructed.

Results Forty-five consecutive patients were included, and 43 of them were included in the final analyses because their rNU specimens were available for reassessment. The only tumour characteristics that were significantly associated with stage were tumour grade $(P<0.001)$, DNA ploidy $(P=0.045)$ and rate of proliferation $(P=0.004)$. No association with stage was noted for size, multifocality or location. Grade, stage and rate of proliferation were associated with DSS.

Conclusions Grade, DNA ploidy and S-phase fraction were the only tumour characteristics associated with stage in our study. However, DNA ploidy was not associated with DSS. The prognostic factors that we identified were tumour grade, stage, and S-phase fraction.
\end{abstract}

Keywords Upper tract urothelial carcinoma · Diagnostics · Staging · Survival · Disease-specific survival · Ureteroscopy · Radical nephroureterectomy

Marianne Brehmer

marianne.brehmer@ki.se

1 Department of Oncology and Pathology, Karolinska Institutet, Stockholm, Sweden

2 Department of Urology, Stockholm South General Hospital, Stockholm, Sweden

3 Division of Urology, Karolinska University Hospital, Stockholm, Sweden

4 Department of Clinical Pathology and Cytology, Karolinska University Hospital Solna, Stockholm, Sweden

5 Division of Urology, Department of Clinical Sciences, Danderyd Hospital, Karolinska Institutet, Stockholm, Sweden

\section{Introduction}

Balancing preservation of renal function against optimisation of oncological treatment in patients with upper tract urothelial carcinoma (UTUC) is challenging, and the guidelines developed by the European Association of Urology (EAU) have addressed this issue by dividing UTUC into high- and low-risk disease [1]. Evidence of renal insufficiency as an independent risk factor for mortality and cardiovascular disease [2] strengthens the incentive to reserve radical nephroureterectomy (rNU) for high-risk patients. Performing rNU on superficial low-grade tumours is actually overtreatment in many cases [3]. Considering diseasespecific survival (DSS), organ-sparing treatment may represent a good option in patients with low-risk UTUC [1, 4-7]. Being able to differentiate between low- and highrisk UTUC is of the utmost importance to aid treatment 
decisions, and accurate risk stratification is crucial in this context. A reliable model for preoperative identification of stage and organ confinement is essential for choice of treatment modality. Preoperative prognostic models based on retrospective data have been proposed for identification of non-organ-confined UTUC [8, 9], and these approaches suggest that tumour grade and hydronephrosis on imaging are key factors predicting invasiveness. However, imaging, even computed tomography urography (CTU), offers insufficient accuracy for staging of UTUC [10, 11]. For optimal diagnostics, radiological investigations should be combined with ureterorenoscopy (URS) and analysis of cytopathological samples [10, 12].

Stage and grade seem to be the most important prognostic factors in UTUC [1, 3, 5, 7, 13, 14]. Direct staging using biopsies is not possible [15], because biopsies must be small and superficial to avoid ureteral perforation and risk of tumour seeding $[16,17]$. Tumour heterogeneity may be an important factor complicating correct grading from small biopsies. This problem persists despite promising novel diagnostic methods such as confocal laser endomicroscopy or optical coherence tomography [18]. Good correlation between grade and stage has been observed in some studies [17, 19].

Regarding grading, voided urine cytology and focal cytology (taken as drip cytology) have been reported to have sensitivity as low as $20 \%$ [20], whereas barbotage cytology has proven to be highly sensitive [21]. Furthermore, several studies have noted that it is difficult to achieve correct grading of endoscopic samples [22, 23], but that diagnostic accuracy can be improved by examining both barbotage cytology and biopsy specimens $[12,21]$. Nevertheless, it is clear that direct staging of UTUC has limitations.

The aim of this prospective study was to investigate UTUC to identify the tumour characteristics that are associated with invasiveness and those that are relevant for DSS.

\section{Patients and methods}

The study was approved by the Regional Ethical Review Board and was performed in accordance with the Declaration of Helsinki. Informed consent was obtained from all patients.

From a prospective consecutive cohort of patients with suspicion of UTUC, those who were diagnosed with UTUC using URS prior to rNU between 2005 and 2012 were included in the present study. Tumour characteristics were analysed for prediction of invasiveness and association with DSS. All rNUs were performed within 1 month after URS. rNU was offered to all patients with UTUC lacking absolute contraindications, as recommended in the EAU guidelines at that time. All patients had M0 disease. The protocol for diagnostic URS and collection of samples has previously been described [21]. In short, at URS, focal barbotage specimens were obtained for cytology and DNA ploidy. Flow cytometry DNA ploidy in endoscopic barbotage samples have previously been proven comparable to ploidy in rNU specimens [21]. Biopsies were taken from suspicious lesions, using Piranha $^{\mathrm{TM}} 3 \mathrm{Ch}$ ureteroscopic biopsy forceps (Boston Scientific Nordic AB, Helsingborg, Sweden).

Tumour size (surface diameter) was visually assessed at URS and also measured in rNU specimens. Tumour stage, grade, ploidy, and rate of proliferation [24] were evaluated in subsequent rNU specimens (paraffin-embedded tissue blocks). We also analysed association with stage for the following parameters: grade, size, multifocality, location, flow cytometry of DNA ploidy, and rate of proliferation (proportion of cells in S-phase of cell cycle). Rate of proliferation was also tested for possible association with tumour grade. Ploidy was categorised as diploid (diploid and tetraploid) or aneuploid (non-tetraploid aneuploid). Surgical technique of rNU varied, one-third of the operations were performed laparoscopically, whereas the others were performed with open surgery. In the majority of the cases the bladder cuff was removed by extravesical open approach.

Stage was categorised as superficial (pTa-1 $[ \pm \mathrm{CIS}]$ or CIS only), or invasive ( $\geq$ pT2 $2 \pm$ CIS). Tumours were graded according to the WHO 1999 classification [25], which is used as the standard in our region, because it is considered to be more useful clinically and also to offer higher resolution and more accurately predict invasiveness compared with the WHO 2004 classification [5, 13]. The TNM/UICC system 2002 [26] was used for tumour staging. Ploidy and rate of proliferation in rNU specimens were determined by flow cytometry. Tumour size of exophytic tumours was categorised by a surface diameter smaller or larger than $20 \mathrm{~mm}$. rNU specimens and focal samples were reassessed by a single specialised pathologist.

DSS in April 2018 was analysed regarding possible association with stage, grade, size, multifocality, location, ploidy and rate of proliferation.

\section{Statistical analysis}

Associations and hazard ratios were tested using Fisher's exact test, Pearson Chi-square or Cox's regression. Kaplan-Meier survival curves were constructed. Ninety-five percent confidence intervals (CIs) were calculated, and a level of 0.05 was considered significant. The statistical analyses were performed using SPSS 23.0 and Microsoft Excel for Mac 2011 (14.3.9). A professional statistician was consulted.

\section{Results}

45 consecutive patients were prospectively included, and 43 of them were included in the final analyses because their rNU specimens were available for reassessment. 26 of these 43 
patients had exophytic superficial tumours, 12 had invasive tumours, and 5 had CIS only. Patient and tumour characteristics are listed in Table 1.

S-phase was statistically significantly different between exophytic superficial and invasive UTUC $(P=0.011)$, as well as between CIS only and invasive tumours $(P=0.011)$. However, no difference was found between exophytic Ta-T1 tumours and CIS, $(P=0.482)$ (Table 1$)$.

A statistically significant association was also found between ploidy and grade $(P<0.001)$ and rate of proliferation (S-phase) and grade (G1: $2.2 \%$ [95\% CI 1.1-3.3], G2: 6.5\% [95\% CI 3.8-9.3], G3: 10.0 [95\% CI 7.6-12.5]). The only tumour characteristics that were significantly associated with stage, superficial (pTa-1, CIS) or invasive (>T1) were tumour grade $(P<0.001)$, DNA ploidy $(P=0.045)$ and rate of proliferation $(P=0.002)$; that is, no association with stage was noted for tumour size, multifocality or location (Table 2).

Grade, stage and rate of proliferation were the only tumour characteristics that were associated with DSS $(P=0.044$, 0.023 and 0.006 , respectively). Associations between DSS and location $(P=0.716)$, multifocality $(P=0.191)$, tumour size $(P=0.719)$, ploidy $(P=0.126)$, and history of bladder cancer $(P=0.866)$ were not statistically significant. At a median follow-up time of 95 months (range 4-144 months), 16/43 patients had died: 10 from urothelial carcinoma and six from other causes. At that time, DSS for all patients was $77 \%$. Stratified by stage, DSS was $88 \%$ for superficial tumours (pTa- $1 \pm$ CIS), $80 \%$ for CIS only, and $50 \%$ for invasive tumours. Stratified by grade, DSS was 100,85 , and $53 \%$ for G1, G2, and exophytic G3, respectively. There was a 6.12 times higher risk of dying in invasive UTUC compared to superficial exophytic UTUC, $P=0.011$. Kaplan-Meier survival curves for stages and grades are shown in Fig. 1. For superficial UTUC (exophytic or CIS) DSS decreased by $25 \%$ for every percent increase in rate of proliferation (S-phase) $(P=0.027)$. The proportion of cells in S-phase was significantly higher in invasive tumours than in superficial $(P=0.002)$ but there was no statistically significant decrease in DSS for increasing rate of proliferation in invasive UTUC $(P=0.969)$.

\section{Discussion}

Grade, DNA ploidy and rate of proliferation (S-phase) were the only parameters that predicted invasiveness, of which grade and rate of proliferation were the strongest. Extra caution should be observed when considering G2 tumours, because it is extremely difficult to predict the stage of such lesions based on endoscopic samples, as noted in our analysis as well as in other studies [8, 17, 19, 22, 27-29]. The proportion of invasive $\mathrm{G} 2$ tumours in the specimens we investigated was $15 \%(2 / 13)$, which is clearly smaller than the proportions of $28-45 \%$ reported by other authors $[6,17$,
$22,27]$. This suggests that $\mathrm{G} 2$ tumours represent a heterogeneous group, and hence a better method is needed to predict invasiveness in this subset. Factors that plausibly affect the mentioned difference are interobserver variability in UTUC pathology evaluation and tumour heterogeneity. Superficial and low-grade tumours are overrepresented in our investigation compared with other reports, which might be explained by the main inclusion criterion we used: patients were to have had URS prior to rNU due to suspicion of UTUC. Some patients with "obvious" findings on imaging were sent straight to rNU during the years covered by our study. Also, our investigation was initiated before organ-sparing treatment was introduced for all patients with what is now defined as low-risk UTUC. Consequently, the present cohort consisted of nearly equal numbers of G1-G3 tumours.

Overall in our study, it was more likely for superficial tumours to be diploid and invasive tumours to be aneuploid. Although there was a strong association between stage and ploidy in general, ploidy could not be used to distinguish between superficial and invasive G2 tumours. The clinical difficulty in this context is illustrated by the following: the two G2 tumours in our material, which would not have been suitable for organ-sparing treatment because they were stage pT2 and pTa + CIS, respectively, were actually diploid. Rate of proliferation was useful in assessing invasiveness, nevertheless, considering the small number of patients with invasive $\mathrm{G} 2$ tumours in our investigation, it is impossible to draw any far-reaching conclusions about G2 disease and/ or rate of proliferation. CIS had a low rate of proliferation, which may be consistent with slow progression of the disease, as also indicated by the only death in a patient with CIS occurring at 79 months after diagnosis. The survival rates in our study are comparable to those reported by Holmäng and Johansson [13].

Rate of proliferation was strongly associated with DSS. Since rate of proliferation can be analysed from URS barbotage samples [21] it may be a valuable tool to predict highrisk UTUC. However, the number of patients in this study are too few to calculate a cut-off value of rate of proliferation for superficial and invasive UTUC for clinical use. Risk of dying in UTUC with increasing rate of proliferation seems to reach a plateau at a level below the range of invasive disease, but this needs further exploration.

Despite a high proportion of both multifocal tumours and tumours $>20 \mathrm{~mm}$ in our material, we found no statistically significant association between stage and multifocality, location, or surface diameter. The lack of correlation between stage and tumour size might be explained by an inappropriate categorisation of size resulting in loss of resolution. In short, size was categorised as CIS or exophytic tumours smaller than or larger than $20 \mathrm{~mm}$, based on risk stratification in the EAU guidelines [1], and 14/26 (54\%) of the superficial tumours in our cohort were larger than $20 \mathrm{~mm}$. 
Table 1 Patient and tumour characteristics of 43 patients who underwent nephroureterectomy after ureterorenoscopy

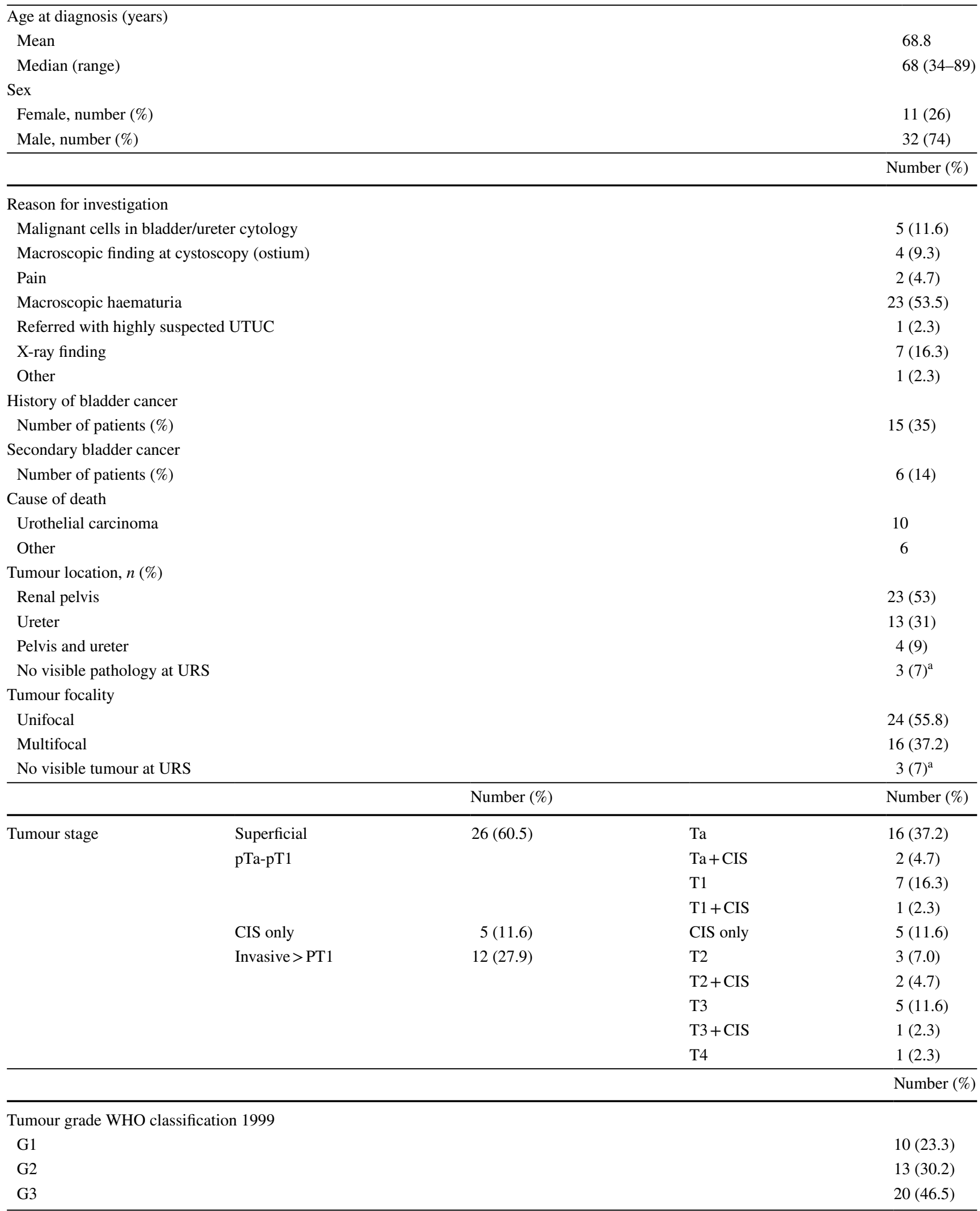


Table 1 (continued)

\begin{tabular}{|c|c|c|}
\hline & & Number $(\%)$ \\
\hline \multicolumn{3}{|l|}{ Tumour surface diameter ${ }^{b}$} \\
\hline$\leq 20 \mathrm{~mm}$ & & $19(44.2)$ \\
\hline$>20 \mathrm{~mm}$ & & $16(37.2)$ \\
\hline CIS only & & $5(11.6)$ \\
\hline \multicolumn{3}{|l|}{ Tumour ploidy } \\
\hline Diploidy (diploid and tetraploid) & & $22(51.2)$ \\
\hline Aneuploidy (non-tetraploid aneuploid) & & $21(48.8)$ \\
\hline \multicolumn{3}{|l|}{ Combined stages and grades number } \\
\hline \multirow[t]{3}{*}{ Superficial (pTa, pT1 \pm CIS) 26} & & G1 10 \\
\hline & & G2 12 \\
\hline & & G3 4 \\
\hline CIS only & & G3 5 \\
\hline \multirow[t]{4}{*}{ Invasive (pT2-4 \pm CIS) 12} & & G1 0 \\
\hline & & $\mathrm{G} 21$ \\
\hline & & G3 11 \\
\hline & Mean, \% $(95 \% \mathrm{CI})$ & Median, \% (range) \\
\hline \multicolumn{3}{|c|}{$\begin{array}{l}\text { Rate of proliferation (proportion of cells in S-phase of the cell cycle), } \\
\text { overall mean } 7.156\end{array}$} \\
\hline Superficial (pTa, pT1 \pm CIS) $N=26$ & $6.0(4.0-8.1)$ & $3.8(0.4-16.6)$ \\
\hline CIS only $N=5$ & $3.3(0.3-6.3)$ & $2.4(1.2-6.7)$ \\
\hline Invasive (pT2-4 \pm CIS) $N=12$ & $11.2(8-14.3)$ & $11.7(2.6-21.8)$ \\
\hline Grade 1 & $2.2(1.1-3.3)$ & $2(0.4-5.7)$ \\
\hline Grade 2 & $6.5(3.8-9.3)$ & $5.4(2.1-16.6)$ \\
\hline Grade 3 & $10.0(7.6-12-5)$ & $11(1.2-21.8)$ \\
\hline
\end{tabular}

${ }^{\mathrm{a}}$ All were CIS only

${ }^{\mathrm{b}}$ Unknown size in three patients

Table 2 Tumour characteristics and their association with stage (pTa-1 and CIS or $>$ T1)

\begin{tabular}{ll}
\hline & Statistical significance \\
\hline Grade & $P<0.001$, significant \\
DNA ploidy & $P=0.045$, significant \\
$\begin{array}{l}\text { Proportion of cells in S-phase of the cell } \\
\text { cycle }\end{array}$ & $P=0.004$, significant \\
Size, surface diameter & $P=0.78$, not significant \\
Multifocality & $P=0.18$, not significant \\
Location of tumour (renal pelvis or ureter) & $P=0.31$, not significant \\
\hline
\end{tabular}

Evidence regarding the role of tumour size is conflicting, and most studies report size in categories and with different cut-off limits, which makes it difficult to compare results. Our findings agree with those obtained by Villa et al. [7], who questioned the usefulness of tumour size but underlined the importance of grade. On the other hand, MilenkovicPetronic et al. [30] used a cut-off of $30 \mathrm{~mm}$ and found a statistically significant association between stage and size.
Some studies have also investigated the impact of size on survival. Notably, both Holmäng and Johansson [13] and Simone et al. [14] observed that larger size was associated with worse outcome, whereas we found no such correlation. Correct grading is more important than a strict size cut-off when selecting treatment modality, because grade is a better predictor of invasion and survival.

\section{Conclusion}

Grade, DNA ploidy and S-phase fraction were the only tumour characteristics associated with stage in our study. However, DNA ploidy was not associated with DSS. The prognostic factors that we identified were tumour grade, stage, and S-phase fraction. Our findings suggest that correct tumour grading plays a crucial role in the diagnostics of UTUC. To further improve preoperative risk stratification, future research should focus on improved methods for correct grading and identification of other reliable markers of 


\section{(a) Stage-stratified DSS for patients with UTUC treated by rNU}

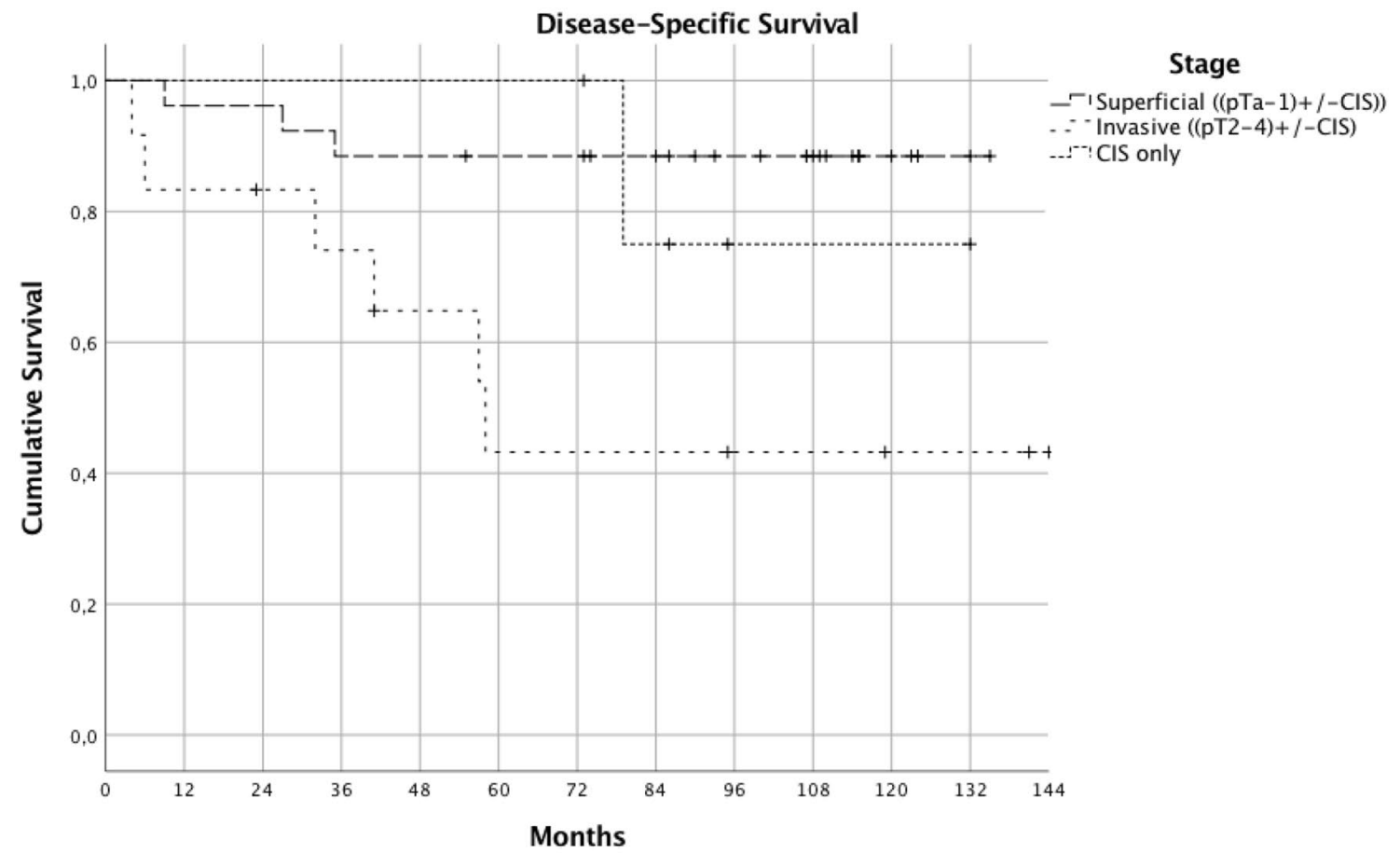

(b) Grade-stratified DSS for patients with UTUC treated by rNU

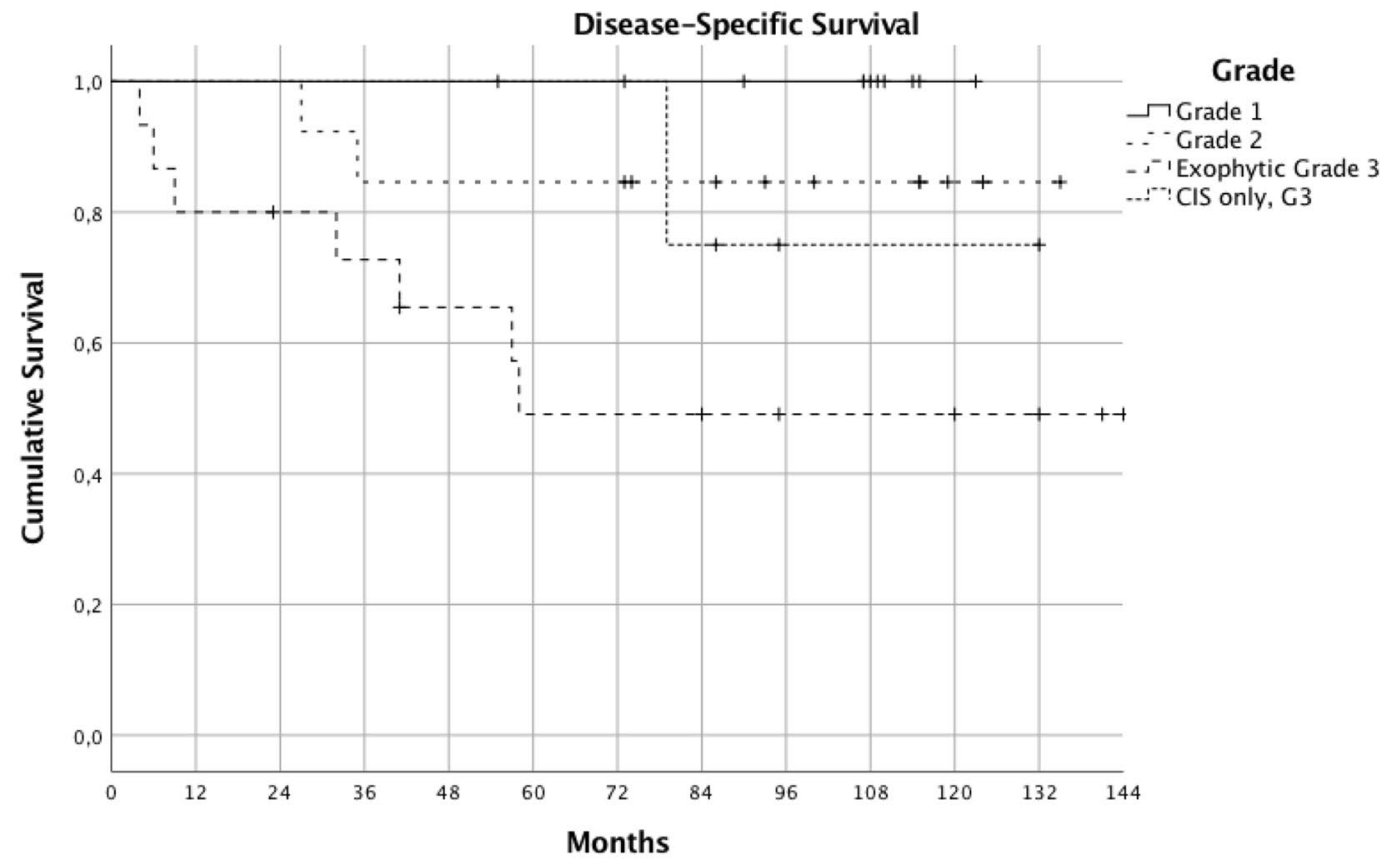

Fig. 1 Kaplan-Meier curves of disease-specific survival stratified by tumour stage and grade. a Stage-stratified DSS for patients with UTUC treated by rNU. Kaplan-Meier curves of disease-specific survival stratified by tumour stage. 5-year disease-specific survival (60 months) was $88 \%$ for superficial tumours, $50 \%$ for invasive tumours, $100 \%$ for CIS only, and $79 \%$ in total. Hazard ratio for DSS in invasive compared with exophytic superficial UTUC was 6.12,
$P=0.011$. Hazard ratio for DSS in exophytic superficial compared to CIS was $1.606, P=0.682$ (not significant). b Grade-stratified DSS for patients with UTUC treated by rNU. Kaplan-Meier curves of diseasespecific survival stratified by tumour grade. 5-year disease-specific survival (60 months) was $100 \%$ for G1, $85 \%$ for G2, 53\% for exophytic G3, $100 \%$ for CIS only and $79 \%$ in total. Overall log rank was 9.618, $P=0.022$ 
high-risk disease, cell proliferation and others, especially with regard to $\mathrm{G} 2$ tumours.

Author contributions CM: study design, data collection, data analysis, and manuscript writing/editing. AG: data collection and manuscript editing. GJ: laboratory work and microscopy and manuscript editing. BT: data analysis and manuscript editing. MB: protocol/project development, data analysis, co-ordination of study, and manuscript editing.

\section{Compliance with ethical standards}

Conflict of interest The authors declare that they have no conflict of interest.

Ethical approval This study was performed according to the $1964 \mathrm{Hel}-$ sinki Declaration and was approved by the regional ethics committee. Informed consent was obtained from all individuals participating in the study.

Open Access This article is distributed under the terms of the Creative Commons Attribution 4.0 International License (http://creativeco mmons.org/licenses/by/4.0/), which permits unrestricted use, distribution, and reproduction in any medium, provided you give appropriate credit to the original author(s) and the source, provide a link to the Creative Commons license, and indicate if changes were made.

\section{References}

1. Rouprêt M, Babjuk M, Compérat E et al (2018) European association of urology guidelines on upper urinary tract urothelial carcinoma: 2017 update. Eur Urol 73:111-122

2. Go AS, Chertow GM, Fan D, McCulloch CE, Hsu C-y (2004) Chronic kidney disease and the risks of death, cardiovascular events, and hospitalization. N Engl J Med 351:1296-1305

3. Grasso M, Fishman AI, Cohen J, Alexander B (2012) Ureteroscopic and extirpative treatment of upper urinary tract urothelial carcinoma: a 15-year comprehensive review of 160 consecutive patients. BJU Int 110:1618-1626

4. Cutress ML, Stewart GD, Zakikhani P, Phipps S, Thomas BG, Tolley DA (2012) Ureteroscopic and percutaneous management of upper tract urothelial carcinoma (UTUC): systematic review. BJU Int 110:614-628

5. Elawdy MM, Taha DE, Elbaset MA, Abouelkheir RT, Osman Y (2016) Histopathologic characteristics of upper tract urothelial carcinoma with an emphasis on their effect on cancer survival: a single-institute experience with 305 patients with long-term follow-up. Clin Genitourin Cancer 14:e609-e615

6. Painter DJ, Denton K, Timoney AG, Keeley FX (2008) Ureteroscopic management of upper-tract urothelial cancer: an exciting nephron-sparing option or an unacceptable risk? J Endourol 22:1237-1239

7. Villa L, Haddad M, Capitanio U et al (2018) Which patients with upper tract urothelial carcinoma can be safely treated with flexible ureteroscopy with holmium:YAG laser photoablation? Long-term results from a high volume institution. J Urol 199:66-73

8. Brien JC, Shariat SF, Herman MP et al (2010) Preoperative hydronephrosis, ureteroscopic biopsy grade and urinary cytology can improve prediction of advanced upper tract urothelial carcinoma. J Urol 184:69-73

9. Favaretto RL, Shariat SF, Savage C et al (2012) Combining imaging and ureteroscopy variables in a preoperative multivariable model for prediction of muscle-invasive and nonorgan confined disease in patients with upper tract urothelial carcinoma. BJU Int 109:77-82

10. Grahn A, Melle-Hannah M, Malm C et al (2017) Diagnostic accuracy of computed tomography urography and visual assessment during ureterorenoscopy in upper tract urothelial carcinoma. BJU Int 119:289-297

11. Wang L-J, Wong Y-C, Chuang C-K, Huang C-C, Pang S-T (2009) Diagnostic accuracy of transitional cell carcinoma on multidetector computerized tomography urography in patients with gross hematuria. J Urol 181:524-531 (discussion 531)

12. Margolin EJ, Matulay JT, Li G et al (2018) Discordance between ureteroscopic biopsy and final pathology for upper tract urothelial carcinoma. J Urol 199:1440-1445

13. Holmäng S, Johansson SL (2005) Urothelial carcinoma of the upper urinary tract: comparison between the WHO/ISUP 1998 consensus classification and WHO 1999 classification system. Urology. 66:274-278

14. Simone G, Papalia R, Loreto A, Leonardo C, Sentinelli S, Gallucci M (2009) Independent prognostic value of tumour diameter and tumour necrosis in upper urinary tract urothelial carcinoma. BJU Int 103:1052-1057

15. Vashistha V, Shabsigh A, Zynger DL (2013) Utility and diagnostic accuracy of ureteroscopic biopsy in upper tract urothelial carcinoma. Arch Pathol Lab Med 137:400-407

16. Hafner C, Knuechel R, Zanardo L et al (2001) Evidence for oligoclonality and tumor spread by intraluminal seeding in multifocal urothelial carcinomas of the upper and lower urinary tract. Oncogene 20:4910-4915

17. Williams SK, Denton KJ, Minervini A et al (2008) Correlation of upper-tract cytology, retrograde pyelography, ureteroscopic appearance, and ureteroscopic biopsy with histologic examination of upper-tract transitional cell carcinoma. J Endourol 22:71-76

18. Bus MT, de Bruin DM, Faber DJ et al (2016) Optical coherence tomography as a tool for in vivo staging and grading of upper urinary tract urothelial carcinoma: a study of diagnostic accuracy. J Urol 196:1749-1755

19. Keeley FX, Kulp DA, Bibbo M, McCue PA, Bagley DH (1997) Diagnostic accuracy of ureteroscopic biopsy in upper tract transitional cell carcinoma. J Urol 157:33-37

20. Messer J, Shariat SF, Brien JC et al (2011) Urinary cytology has a poor performance for predicting invasive or high-grade upper-tract urothelial carcinoma. BJU Int 108:701-705

21. Malm C, Grahn A, Jaremko G, Tribukait B, Brehmer M (2017) Diagnostic accuracy of upper tract urothelial carcinoma: how samples are collected matters. Scand J Urol 51:137-145

22. Skolarikos A, Griffiths TRL, Powell PH, Thomas DJ, Neal DE, Kelly JD (2003) Cytologic analysis of ureteral washings is informative in patients with grade 2 upper tract TCC considering endoscopic treatment. Urology 61:1146-1150

23. Wang JK, Tollefson MK, Krambeck AE, Trost LW, Thompson RH (2012) High rate of pathologic upgrading at nephroureterectomy for upper tract urothelial carcinoma. Urology. 79:615-619

24. Heiden T, Wang N, Tribukait B (1991) An improved Hedley method for preparation of paraffin-embedded tissues for flow cytometric analysis of ploidy and S-phase. Cytometry 12:614-621

25. Mostofi FK, Davis CJ, Sesterhenn IA (1999) WHO histologic typing of urinary bladder tumors. Springer, Berlin

26. TNM classification of malignant tumours UICC (2002) International union against cancer. Wiley-Liss, New York

27. Brown GA, Matin SF, Busby JE et al (2007) Ability of clinical grade to predict final pathologic stage in upper urinary tract transitional cell carcinoma: implications for therapy. Urology. 70:252-256 
28. Guarnizo E, Pavlovich CP, Seiba M, Carlson DL, Vaughan ED, Sosa RE (2000) Ureteroscopic biopsy of upper tract urothelial carcinoma: improved diagnostic accuracy and histopathological considerations using a multi-biopsy approach. J Urol 163:52-55

29. Shiraishi K, Eguchi S, Mohri J, Kamiryo Y (2003) Role of ureteroscopic biopsy in the management of upper urinary tract malignancy. Int J Urol 10:627-630
30. Milenkovic-Petronic D, Milojevic B, Djokic M et al (2014) The impact of tumor size on outcomes in patients with upper urinary tract urothelial carcinoma. Int Urol Nephrol 46:563-569

Publisher's Note Springer Nature remains neutral with regard to jurisdictional claims in published maps and institutional affiliations. 